\title{
La violence sexuelle envers les enfants est-elle en baisse au Canada? Résultats d'enquêtes rétrospectives représentatives à l'échelle nationale
}

\author{
M. Shields; L. Tonmyr, Ph. D.; W. Hovdestad, Ph. D.
}

Cet article a fait l'objet d'une évaluation par les pairs.

Diffuser cet article sur Twitter

\section{Résumé}

Introduction : Selon de nombreuses sources de données, la violence sexuelle envers les enfants (VSE) est en baisse aux États-Unis depuis le début des années 1990. Il semble par ailleurs qu'elle ait connu une hausse après la Seconde Guerre mondiale. Cette étude a pour but de faire l'examen des estimations de prévalence des cas de violence sexuelle durant l'enfance (VSdE) signalés rétrospectivement dans le cadre de deux enquêtes nationales représentatives menées auprès de la population canadienne.

Méthodologie : Les données proviennent de 13931 répondants de 18 à 76 ans interrogés dans le cadre de l'enquête GENACIS (Gender, Alcohol and Culture: An International Study) - Canada de 2004-2005 et de 22169 résidents âgés de 18 ans et plus ayant participé à l'Enquête sur la santé dans les collectivités canadiennes - Santé mentale (ESCC - Santé mentale) de 2012. Nous présentons des comparaisons de la prévalence de la VSdE par sexe et par groupe d'âge à la fois entre ces deux enquêtes et au sein de chacune.

Résultats : Les résultats des deux enquêtes indiquent une baisse de la VSE depuis 1993, qui va de pair avec la baisse observée aux États-Unis. Il ressort également des résultats que le risque de VSE était plus élevé entre 1946 et 1992 qu’avant 1946. Les données sont plus solides pour les femmes.

Conclusion : Constater une baisse de la VSE au Canada depuis le début des années 1990 est encourageant, étant donné les effets débilitants à long terme qui sont associés à la VSE. Une surveillance continue est essentielle. Ces effets négatifs à long terme sont la preuve de l'importance de poursuivre sur cette lancée, pour passer d'un risque faible à un risque nul.

Mots-clés : violence envers les enfants, mauvais traitements à l'égard des enfants, violence sexuelle durant l'enfance, prévalence, enfance, année-personne, temps de passage, cohorte

\section{Introduction}

L'incidence de la violence sexuelle envers les enfants (VSE), responsable de troubles corollaires invalidants tout au long de la vie, est sans équivoque ${ }^{1,2}$. Son ampleur et l'évolution de sa prévalence au fil du temps sont moins évidentes. Une grande partie de l'information accessible sur l'incidence de la VSE provient de données recueillies par les professionnels de la protection de l'enfance.
Aux États-Unis, les données provenant des professionnels et des organismes de protection de l'enfance qui œuvrent auprès d'enfants dans les écoles, les établissements hospitaliers et les garderies font état d'une hausse du nombre de cas de VSE entre le milieu des années 1970 et le début des années $1990^{3-5}$. D'aucuns estiment que la hausse observée dans les données compilées par ces organismes pendant les années 1970 et 1980 traduit, en réalité, la

\section{Points saillants}

- Les données de deux enquêtes représentatives de la population canadienne à l'échelle nationale font état d'une baisse de la violence envers les enfants (VSE) depuis 1993, qui va de pair avec la baisse observée aux États-Unis.

- Il ressort également des résultats que le risque de VSE était plus élevé entre 1946 et 1992 qu'avant 1946.

- Les données sont plus solides pour les femmes.

- Malgré leur caractère rétrospectif, les enquêtes basées sur une population peuvent - si elles sont méthodologiquement cohérentes - dégager les tendances de la VSE, ce qui permet de dresser un portrait plus complet des données contemporaines provenant d'enquêtes sur les enfants.

- Les effets négatifs à long terme qui sont associés à la VSE démontrent l'importance de poursuivre sur cette lancée pour l'éliminer complètement. Assurer une surveillance continue est indispensable - aucun laisser-aller n’est tolérable.

hausse de la sensibilisation du public et les modifications apportées aux mécanismes d'identification et de signalement de la VSE plutôt qu'une réelle croissance de son incidence $^{3}$. En parallèle, les données provenant du National Child Abuse and Neglect Data System des États-Unis indiquent qu'entre 1992 et 2013, la VSE a diminué de $64 \%$, à l'instar du viol par contrainte, qui enregistre également une baisse dans les données statistiques du Federal Bureau of Investigation $(\mathrm{FBI})^{6}$. Cette baisse est d'ailleurs mieux étayée que la hausse précédente, car

Rattachement des auteurs :

Agence de la santé publique du Canada, Ottawa (Ontario), Canada

Correspondance : Lil Tonmyr, Agence de la santé publique du Canada, 785, avenue Carling, IA 6807B, Ottawa (Ontario) K1A 0K9; tél. : 613-240-6334; téléc. : 613-960-0944; courriel : Lil.Tonmyr@phac-aspc.gc.ca 
elle ne survient pas à la suite de l'adoption de nouvelles procédures administratives? Elle coïncide avec des améliorations dans d'autres sphères du bien-être de l'enfant aux États-Unis au cours de cette période et avec une baisse des grossesses chez les adolescentes, du suicide chez les adolescents et de la prévalence de la pauvreté infantile ${ }^{8-10}$

L'un des grands obstacles à l'étude des tendances de l'incidence réelle de la VSE à partir de données provenant des organismes de protection de l'enfance et des autorités policières est que les estimations tirées de ces sources ne constituent que la pointe de l'iceberg. En effet, les cas de violence sexuelle ne sont généralement pas signalés à ces autorités ${ }^{11,12}$. À défaut d'enquêtes directes auprès des enfants et des adolescents, les enquêtes rétrospectives sur la population, dans le cadre desquelles les chercheurs interrogent les adultes et les adolescents sur leurs expériences dans l'enfance, offrent une autre source d'information permettant d'obtenir des estimations plus précises sur la $\mathrm{VSE}^{3}$. Nous employons l'expression « violence sexuelle durant l'enfance » (VSdE) pour désigner les agressions sexuelles survenues au cours de l'enfance et mentionnées lors de ces études.

Les données tirées de nombreuses enquêtes sur la victimisation aux États-Unis font état d'une diminution du nombre de cas de VSE depuis les années 1990, ce que reflètent les tendances observées à la lumière des données provenant des organismes de protection de l'enfance ${ }^{13}$. D'autres données d'enquête indiquent une hausse de la VSE après la Seconde Guerre mondiale ${ }^{3,14-16}$. Certains chercheurs ${ }^{17,18}$ estiment que ce sont plutôt les lois, le climat social, les écarts dans les taux de réponse et les différentes définitions de la VSdE qui expliquent cette augmentation apparente.

Deux études récemment menées au Canada auprès de la population adulte générale ont comporté des questions sur la VSdE, soit l'Enquête sur la santé dans les collectivités canadiennes - Santé mentale (ESCC - Santé mentale) de $2012^{19}$ et l'enquête Gender, Alcohol and Culture: An International Study (GENACIS) - Canada menée en 2004-2005 20,21. Nous avons comparé les estimations sur la prévalence de la VSdE (par groupe d'âge) entre ces deux enquêtes et au sein de chacune d'elles. Nous avons évalué la cohérence des données du Canada par rapport aux baisses de la VSE rapportées par Finkelhor et ses collaborateurs ${ }^{6}$ et par rapport aux hausses signalées après la Seconde Guerre mondiale. Nous avons étudié les données provenant des enquêtes canadiennes afin d'y déceler plus spécifiquement des correspondances avec les hypothèses selon lesquelles la VSE était relativement faible avant la fin de la Seconde Guerre mondiale, relativement élevée après la Seconde Guerre mondiale puis relativement faible à partir des années 1990.

\section{Méthodologie}

\section{Sources de données}

Nous nous sommes servis des données de l'enquête GENACIS pour le Canada pour obtenir des estimations sur la VSdE pour 2004-2005. Cette enquête s'inscrivait dans une vaste collaboration internationale et multidisciplinaire sur un éventail de sujets touchant les problèmes liés au genre, à la culture et à l'alcool. Pour le volet canadien de l'enquête GENACIS, les chercheurs ont sélectionné leur échantillon par enquête téléphonique à composition aléatoire afin d'obtenir une portion représentative de la population canadienne âgée de 18 à 76 ans vivant à domicile dans les 10 provinces en $2004-2005^{20}$. Les entrevues ont eu lieu par téléphone au moyen de la technique d'interview téléphonique assistée par ordinateur. L'enquête excluait les ménages n'ayant pas de ligne téléphonique résidentielle, les personnes ne parlant ni l'anglais ni le français et les personnes vivant en établissement ${ }^{21}$. Le taux de réponse s'est élevé à 52,8\% (le nombre de participants ayant répondu au sondage divisé par le nombre total estimatif de ménages admissibles) ${ }^{20}$, soit un échantillon de 14063 répondants (6 009 hommes et 8054 femmes). Notre étude exclut les participants n'ayant pas répondu aux questions sur la VSE (51 hommes et 81 femmes), ce qui réduit la taille de notre échantillon à 13931 répondants (5 958 hommes et 7973 femmes).

Nous avons puisé les estimations sur la prévalence de la VSE pour 2012 dans les données de l'ESCC - Santé mentale. Pour effectuer cette enquête, Statistique Canada a fait appel à une méthode d'échantillonnage en grappes stratifiée à plusieurs degrés ${ }^{19}$. L'ESCC - Santé mentale ciblait les résidents âgés de 15 ans et plus dans les 10 provinces canadiennes, et la majorité (87 \%) des entrevues ont été menées en personne. L'ESCC - Santé mentale excluait les personnes habitant sur les réserves et dans les autres lieux de peuplement autochtone, les membres à temps plein des Forces canadiennes et les personnes en établissement, soit environ $3 \%$ de la population cible. Le taux de réponse a été de $68,9 \%{ }^{19}$, soit 25113 personnes âgées de 15 ans et plus. Les responsables de l'ESCC - Santé mentale ont demandé aux répondants la permission de transmettre les réponses aux partenaires de Statistique Canada, dont l'Agence de la santé publique du Canada, et la plupart des répondants ( $n=23$ 709; $94 \%$ ) leur ont accordé cette permission. Notre étude repose donc sur les données provenant de ces fichiers dits " partagés ». Les questions sur la VSE ont été posées uniquement aux répondants âgés de 18 ans et plus $(n=22486)$. Les répondants ayant refusé de répondre (159 hommes et 158 femmes) ont été exclus de l'étude, ce qui porte notre échantillon final à 22169 répondants $(9910$ hommes et 12259 femmes).

\section{Mesures}

Nous avons placé dans la catégorie des personnes ayant connu de la VSdE les répondants à l'enquête GENACIS ayant répondu par l'affirmative aux questions suivantes :

- « Avant que vous ayez atteint l'âge de 16 ans, est-ce qu'un membre de VOTRE FAMILLE a essayé de vous faire faire ou de vous faire regarder des actes sexuels alors que vous n'étiez PAS consentant? » [traduction]

- " Avant que vous ayez atteint l'âge de 16 ans, est-ce qu'une personne AUTRE QU'UN MEMBRE DE VOTRE FAMILLE a essayé de vous faire faire ou de vous faire regarder des actes sexuels alors que vous n'étiez PAS consentant? » [traduction]

Les répondants de l'ESCC - Santé mentale ont été interrogés à propos de ce qui a pu leur arriver à l'école, dans leur quartier ou dans leur famille avant l'âge de 16 ans :

- «Combien de fois un adulte vous a-t-il forcé, ou essayé de vous forcer, à avoir une activité sexuelle non désirée en vous menaçant, en vous immobilisant ou en vous faisant mal d'une autre façon? »

- «Combien de fois un adulte vous a-t-il touché contre votre volonté d'une manière sexuelle? Par cela je veux dire tout ce qui va d'un attouchement non désiré à un baiser ou des caresses. » 
Les participants de l'ESCC - Santé mentale qui ont répondu avoir vécu ce genre d'expérience au moins une fois ont été classés comme ayant connu de la VSdE.

\section{Analyses}

Nous avons employé deux méthodes pour examiner l'évolution de la prévalence de la VSE au fil du temps. Dans un premier temps, nous avons comparé les estimations de prévalence de la VSdE au sein des mêmes groupes d'âge dans l'enquête GENACIS et dans l'ESCC - Santé mentale. Dans un second temps, nous avons comparé la prévalence de la VSdE entre les différents groupes d'âge au sein de chaque enquête. La comparaison des estimations de prévalence par groupe d'âge est une approche couramment utilisée dans le cadre d'enquêtes rétrospectives chez les adultes afin d'examiner les variables en matière de VSdE en fonction des générations ${ }^{3,22}$. Cette approche réduit les risques que les différences visibles dans la prévalence de la VSdE découlent de différences méthodologiques.

Notre hypothèse était que la prévalence serait relativement plus élevée chez les personnes ayant vécu leur enfance (ayant entre 0 et 15 ans) entre 1946 et 1992 et, à l'inverse, qu'elle serait relativement faible chez les personnes ayant vécu leur enfance avant la fin de la Seconde Guerre mondiale (avant 1946) ou après le début des années 1990 (à partir de 1993). Nous avons utilisé une version modifiée de l'approche conventionnelle de l'année-personne pour créer l'unité d'analyse " année-enfant ». Il s'agit d'une unité de temps individuelle qui désigne l'époque à laquelle la population à l'étude, alors âgée de 0 à 15 ans, a été potentiellement exposée à de la VSE. Nous avons calculé le pourcentage d'années d'enfance que les répondants de chaque groupe d'âge ont vécu au cours des périodes de risque accru et de risque moindre. Le tableau 1 présente le nombre et le pourcentage d'années-enfants (c.-à-d. de 0 à 15 ans) que chaque groupe d'âge (au moment de l'étude) a vécus au cours des trois grandes périodes. La figure 1 est une représentation graphique de la manière dont nous nous y sommes pris pour calculer les pourcentages d'années-enfants pour la période dite à risque accru.

Le tableau 1 illustre les calculs des annéesenfants. Penchons-nous sur le groupe d'âge de 18 à 24 ans sur la première ligne du tableau 1. Ces répondants cumulent un total de 112 années-enfants (les 16 années d'enfance vécues de 0 à 15 ans multipliées par les 7 âges du groupe de 18 à 24 ans, en supposant que les 7 âges y soient également représentés). Dans ce groupe d’âge, les répondants de l'enquête GENACIS ont vécu 70 années-enfants entre 1946 et 1992 , la période à risque accru. Les répondants âgés de 18 ans (nés en 1986) ont vécu 7 années-enfants au cours de cette période (de 1986 à 1992), ceux de 19 ans, 8 annéesenfants, ceux de 20 ans, 9, et ainsi de suite jusqu'au dernier groupe, 24 ans, dont les répondants ont vécu 13 années pendant la période de risque accru, ce qui nous conduit à notre total de $70(7+8+9+10+$ $11+12+13)$.

Ainsi, les répondants du groupe d'âge le plus jeune de l'enquête GENACIS ont vécu 70 de leurs 112 années-enfants (63 \%) au cours de la période que nous supposons à risque accru et les autres années (38 \%) au cours de la période que nous supposons à risque moindre (à partir de 1993). Dans la figure 1 , chaque case ombrée représente une année-enfant vécue pendant la période à risque accru. Chez les répondants de l'enquête GENACIS âgés de 18 à 24 ans, on compte donc 70 cases ombrées (risque accru) sur un total de 112 années-enfants. Les 42 cases restantes supposées apparaître à la droite de la période à risque moindre (à partir de 1993) ne sont pas représentées dans la figure. Les répondants âgés de 35 à 49 ans ont passé l'ensemble de leur 240 années-enfants dans la période à risque accru (de 1946 à 1992) et les répondants des groupes de 25 à 34 ans et de 50 à 64 ans ont passé presque toutes leurs annéesenfants pendant cette période (respectivement

TABLEAU 1

Nombre et pourcentage d'années-enfants (0 à 15 ans) vécues au cours de diverses périodes de risque de VSE (1993 à aujourd'hui, 1946 à 1992, avant 1945) parmi les répondants de l'enquête GENACIS et de l'ESCC-Santé mentale, par groupe d'âge au moment des enquêtes

\begin{tabular}{|c|c|c|c|c|c|c|c|c|c|c|c|c|c|c|c|}
\hline \multirow{4}{*}{$\begin{array}{l}\text { Groupe } \\
\text { d'âgé } \\
\text { (ans) }\end{array}$} & \multirow{4}{*}{$\begin{array}{l}\text { Total des } \\
\text { années- } \\
\text { enfants } \\
\text { (0 à } 15 \text { ans) }\end{array}$} & \multicolumn{7}{|c|}{ Enquête GENACIS de 2004} & \multicolumn{7}{|c|}{ ESCC-Santé mentale de 2012} \\
\hline & & \multirow{3}{*}{$\begin{array}{l}\text { Année de } \\
\text { naissance }\end{array}$} & \multicolumn{6}{|c|}{$\begin{array}{l}\text { Nombre et pourcentage (\%) } \\
\text { d'années-enfants (de } 0 \text { à } 15 \text { ans) vécues }\end{array}$} & \multirow{3}{*}{$\begin{array}{l}\text { Année de } \\
\text { naissance }\end{array}$} & \multicolumn{6}{|c|}{$\begin{array}{l}\text { Nombre et pourcentage (\%) } \\
\text { d'années-enfants ( } 0 \text { à } 15 \text { ans) vécues }\end{array}$} \\
\hline & & & \multicolumn{2}{|c|}{$\begin{array}{l}\text { de } 1993 \text { à } \\
\text { aujourd'hui }\end{array}$} & \multicolumn{2}{|c|}{$\begin{array}{l}\text { de } 1946 \\
\text { à } 1992\end{array}$} & \multicolumn{2}{|c|}{ avant 1945} & & \multicolumn{2}{|c|}{$\begin{array}{l}\text { de } 1993 \text { à } \\
\text { aujourd'hui }\end{array}$} & \multicolumn{2}{|c|}{$\begin{array}{l}\text { de } 1946 \\
\text { à } 1992\end{array}$} & \multicolumn{2}{|c|}{ avant 1945} \\
\hline & & & Nombre & $\%$ & Nombre & $\%$ & Nombre & $\%$ & & Nombre & $\%$ & Nombre & $\%$ & Nombre & $\%$ \\
\hline 18 à 24 & 112 & $1980-1986$ & 42 & 38 & 70 & 63 & 0 & 0 & 1988-1994 & 97 & 87 & 15 & 13 & 0 & 0 \\
\hline 35 à 49 & 240 & $1955-1969$ & 0 & 0 & 240 & 100 & 0 & 0 & 1963-1977 & 0 & 0 & 240 & 100 & 0 & 0 \\
\hline 50 à 64 & 240 & $1940-1954$ & 0 & 0 & 219 & 91 & 21 & 9 & 1948-1962 & 0 & 0 & 240 & 100 & 0 & 0 \\
\hline 65 à 76 & 192 & 1928-1939 & 0 & 0 & 45 & 23 & 147 & 77 & 1936-1947 & 0 & 0 & 137 & 71 & 55 & 29 \\
\hline 77 et plus ${ }^{b}$ & 368 & & & & & & & & 1935 ou avant & 0 & 0 & 15 & 4 & 353 & 96 \\
\hline
\end{tabular}

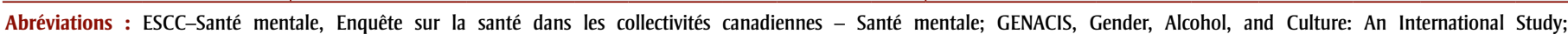
VSE, violence sexuelle envers les enfants.

Remarque : Nous indiquons l'année 2004 pour l'enquête GENACIS, puisque la majorité (93\%) des entrevues auprès des répondants ont été réalisées en 2004.

${ }^{a}$ Total des années-enfants $=($ nombre d'années de naissance dans une cohorte $) \times 16$.

${ }^{\text {b }}$ Nous supposons que l'âge maximal de cette cohorte est de 99 ans. 


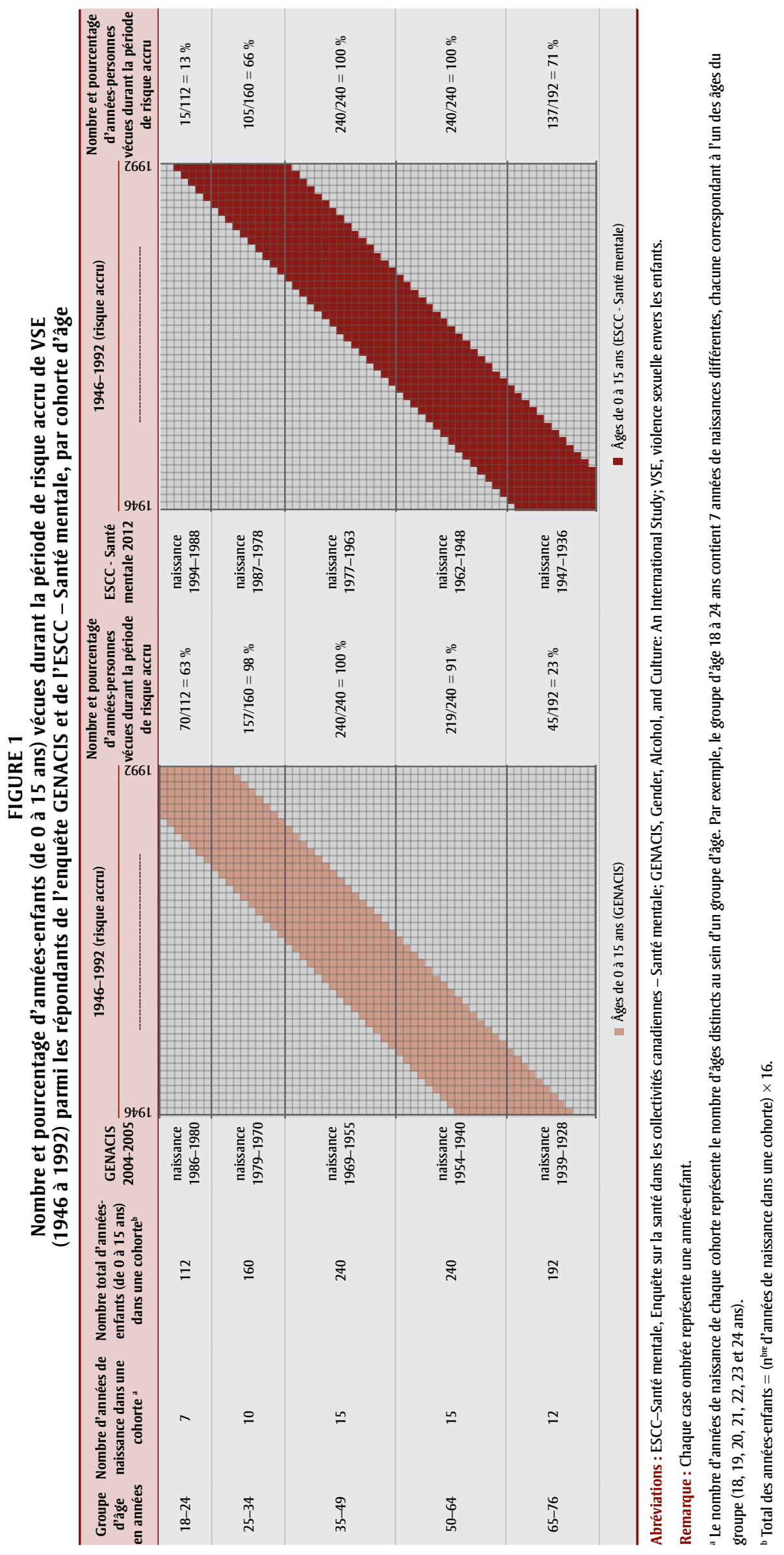

$98 \%$ et $91 \%$ ). Les répondants du groupe le plus âgé (de 65 à 76 ans) ont vécu la plupart de leurs années-enfants avant la fin de la Seconde Guerre mondiale $(147 / 192=77 \%)$, une période à risque moindre selon nos hypothèses. Sur ce point, notons que, sur la figure 1, seules 45 cases ombrées apparaissent dans la période à risque accru pour ce groupe d'âge, les 147 années-enfants restantes de ce groupe étant censées apparaître à gauche, dans la période à faible risque (avant 1946). Ces années-enfants font bien partie des analyses (pour la période à risque moindre) mais elles n'ont pas été intégrées à la figure.

Étant donné que l'ESCC - Santé mentale s'est déroulée 8 ans après l'enquête GENACIS, les répondants dans les groupes d'âge utilisés pour classer les répondants GENACIS auraient vécu des années différentes de leur enfance dans des périodes à risque élevé ou faible. Les quatre groupes d'âge intermédiaires de l'ESCC - Santé mentale $(25$ à 34,35 à 49,50 à 65 et 65 à 76 ans) ont vécu la majorité de leurs années-enfants au cours de la période dite à risque accru. Les répondants des groupes d'âge de 35 à 49 ans et de 50 à 64 ans ont vécu toutes leurs années-enfants au cours de cette période. Pour ce qui est du groupe d'âge le plus jeune (18 à 24 ans) et du groupe d'âge le plus avancé (77 ans et plus), les répondants ont vécu la majorité de leurs années-enfants au cours des périodes dites à moindre risque : les répondants du groupe d'âge le plus jeune ont vécu $87 \%$ de leurs années-enfants après 1993 , et ceux du groupe d'âge le plus avancé ont vécu $96 \%$ de leurs annéesenfants avant la fin de la Seconde Guerre mondiale.

Si on se fie aux estimations de prévalence de la VSdE entre l'enquête GENACIS et l'ESCC - Santé mentale, la notion de VSE semble la même dans les deux enquêtes. Les estimations peuvent néanmoins varier en fonction soit des différences méthodologiques entre les enquêtes soit d'une évolution dans la volonté des participants de dévoiler la VSdE vécue. Pour évaluer les différences méthodologiques susceptibles de jouer sur les estimations, nous avons jumelé les cohortes ayant la même année de naissance dans les deux enquêtes afin de comparer les estimations. Compte tenu de l'intervalle de 8 ans entre les deux enquêtes, nous avons présumé que la prévalence de la VSE serait sensiblement dans les mêmes proportions statistiques 
entre, par exemple, les répondants de l'enquête GENACIS âgés de 18 à 24 ans et les répondants de l'ESCC - Santé mentale âgés de 26 à 32 ans (tous nés entre 1980 et 1986), ce qui constitue une manière d'exclure les différences découlant des autres facteurs.

Nous avons effectué nos analyses à l'aide de la version 5.1 de SAS Enterprise Guide (SAS Institute Inc, Cary, Caroline du Nord, États-Unis). Toutes les estimations reposent sur des données pondérées. La vérification des écarts importants entre l'enquête GENACIS et l'ESCC - Santé mentale a été réalisée à l'aide de tests $t$ totalisés. Pour ce qui est des estimations provenant de l'ESCC - Santé mentale, la méthode bootstrap a servi à calculer la variance et les intervalles de confiance (IC) à $95 \%$ pour bien tenir compte du plan d'enquête complexe de l'ESCC - Santé mentale ${ }^{19}$. Quant aux estimations de l'enquête GENACIS, la variance et les IC à $95 \%$ ont été calculés en suivant l'hypothèse d'un échantillonnage aléatoire simple.

\section{Résultats}

Les estimations de prévalence de la VSdE tirées de l'enquête GENACIS et de l'ESCC Santé mentale se sont révélées similaires chez les 18 à 76 ans, soit 9,8\% d'après l'enquête GENACIS de 2004-2005 et 10,5 \% d'après l'ESCC - Santé mentale de 2012 (tableau 2). Nous constatons néanmoins

TABLEAU 2

Prévalence de la VSdE par sexe et par groupe d'âge au moment des enquêtes menées auprès de la population canadienne à domicile âgée de 18 ans et plus, 2004-2005 et 2012

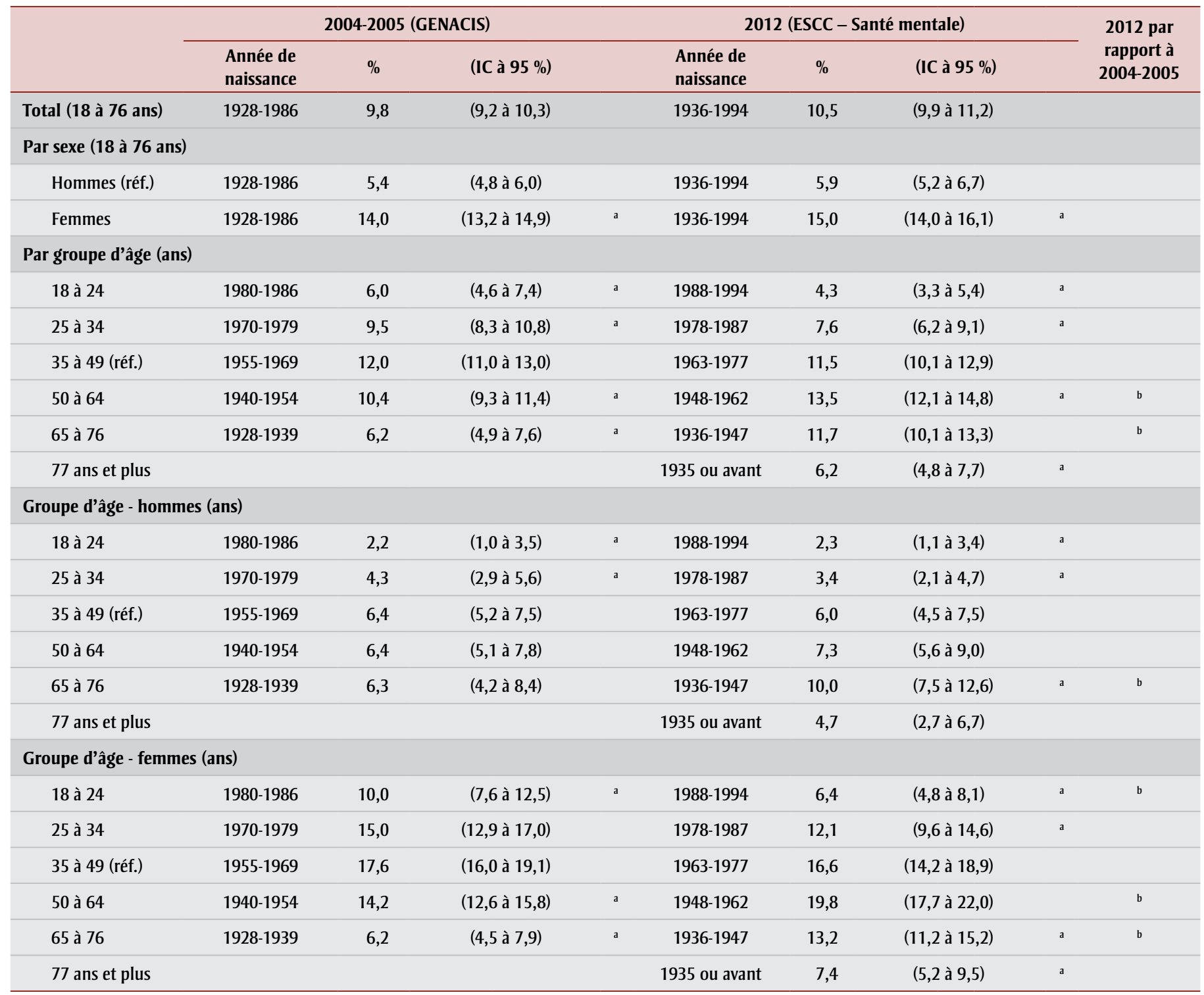

Sources : GENACIS, enquête canadienne de 2004-2005; Statistique Canada, ESCC-SM, 2012 (fichier partagé).

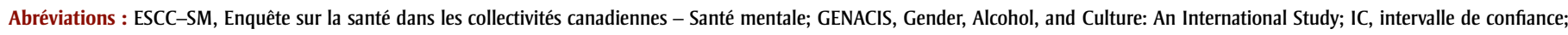
réf., groupe de référence; VSdE, violence sexuelle durant l'enfance.

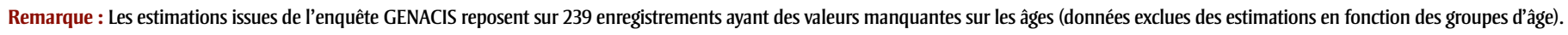

${ }^{a}$ Écart notable par rapport au groupe de référence.

${ }^{\mathrm{b}}$ Écart notable par rapport aux données de 2004-2005. 
certaines différences entre les deux périodes selon les sexes et les groupes d'âge.

Chez les femmes du groupe d'âge le plus jeune (de 18 à 24 ans), la prévalence est passée de $10,0 \%$ en 2004-2005 à 6,4 \% en 2012. Chez les femmes de 25 à 34 ans, la prévalence était de 15,0 \% en 2004-2005 et de $12,1 \%$ en 2012, mais cette baisse n'a pas atteint tout à fait le seuil de signification statistique $(p=0,08)$. Les femmes se trouvant dans ces deux groupes d'âge au moment de l'ESCC - Santé mentale auraient ainsi vécu une plus grande portion de leur enfance après 1993 (période à risque moindre) que celles se trouvant dans ces mêmes groupes d'âge au moment de l'enquête GENACIS.

Inversement, chez les femmes faisant partie des deux derniers groupes d'âge, la prévalence augmente entre les deux périodes : elle passe de $14,2 \%$ à 19,8 \% chez les 50 à 64 ans et de $6,2 \%$ à 13,2\% chez les 65 à 76 ans. Les femmes présentes dans ces groupes d'âge au moment de l'ESCC Santé mentale ont passé une plus grande portion de leur enfance entre 1946 et 1992 (période à risque accru), que les femmes de ces groupes d'âge au moment de l'enquête GENACIS.

Nous avons constaté chez les femmes une association en forme de « $U$ » entre l'âge et la VSdE. En 2004-2005, la prévalence de la VSdE était plus élevée chez les répondantes de 35 à 49 ans (celles qui ont passé toute leur enfance entre 1946 et 1992, la période à risque accru). En 2012, la prévalence la plus élevée s'observait chez les répondantes âgées de 35 à 49 ans et de 50 à 64 ans (qui ont, elles aussi, vécu toute leur enfance pendant cette période à risque accru). Aux deux dates, nous avons observé les estimations de prévalence les plus faibles dans le groupe d'âge le plus jeune et dans le plus avancé. De fait, les répondantes du groupe d'âge plus jeune ont vécu la majeure partie de leur enfance après 1993, et celles du groupe d'âge le plus avancé l'ont vécue avant la fin de la Seconde Guerre mondiale (deux périodes où le risque était moindre).

Chez les hommes, la seule différence majeure entre les enquêtes touche les répondants de 65 à 76 ans, chez qui la prévalence est passée de 6,3 \% en 2004-2005 à 10,0 \% en 2012. Les hommes qui étaient âgés de 65 à 76 ans en 2012 ont vécu $71 \%$ de leur enfance entre 1946 et 1992, période où le risque était accru, tandis que cette situation ne touchait que $23 \%$ des hommes de ce groupe d'âge au moment de l'enquête GENACIS.

Chez les hommes en 2012, la prévalence de la VSdE la plus élevée $(10,0 \%)$ s'observait chez les hommes âgés de 65 à 76 ans, et la plus faible pour les répondants des groupes d'âge les plus jeunes, qui ont passé la majeure partie de leur enfance après 1993, une période où le risque était moindre.

Les comparaisons entre les deux enquêtes pour les cohortes ayant la même année de naissance (tableau 3) présentent des estimations statistiques semblables pour la majorité des groupes formés selon l'année de naissance (ce qui était à prévoir, hormis les différences méthodologiques entre les deux enquêtes). Nous avons cependant constaté des différences importantes entre les hommes nés entre 1940 et 1954 et les

TABLEAU 3

Prévalence de la VSdE par sexe et par cohorte selon l'année de naissance, population à domicile âgée de 18 ans à 76 ans, Canada, 2004-2005 et 2012

\begin{tabular}{|c|c|c|c|c|c|c|c|}
\hline \multirow[b]{2}{*}{$\begin{array}{l}\text { Cohorte par } \\
\text { année de } \\
\text { naissance }\end{array}$} & \multicolumn{3}{|c|}{ 2004-2005 (GENACIS) } & \multicolumn{3}{|c|}{2012 (ESCC - Santé mentale) } & \multirow[b]{2}{*}{$\begin{array}{l}2012 \text { par } \\
\text { rapport à } \\
2004-2005\end{array}$} \\
\hline & $\begin{array}{l}\text { Groupe d'âge au } \\
\text { moment de l'enquête } \\
\text { GENACIS (ans) }\end{array}$ & $\begin{array}{l}\text { \% de VSdE } \\
\text { signalée }\end{array}$ & (IC à $95 \%)$ & $\begin{array}{l}\text { Groupe d'âge au } \\
\text { moment de } \\
\text { l'ESCC (ans) }\end{array}$ & $\begin{array}{l}\text { \% de VSdE } \\
\text { signalée }\end{array}$ & (IC à $95 \%)$ & \\
\hline & Hommes & & & Hommes & & & \\
\hline $1980-1986$ & 18 à 24 & 2,2 & $(1,0$ à 3,5$)$ & 26 à 32 & 2,5 & $(1,1$ à 3,8$)$ & \\
\hline 1955-1969 & 35 à 49 & 6,4 & (5,2 à 7,5) & 43 à 57 & 6,9 & (5,3 à 8,5) & \\
\hline $1940-1954$ & 50 à 64 & 6,4 & (5,1 à 7,8) & 58 à 72 & 9,1 & $(7,0$ à 11,1$)$ & a \\
\hline \multirow[t]{2}{*}{ 1928-1939 } & 65 à 76 & 6,3 & $(4,2$ à 8,4$)$ & 73 à 84 & 5,1 & (3,4 à 6,9) & \\
\hline & Femmes & & & Femmes & & & \\
\hline $1955-1969$ & 35 à 49 & 17,6 & $(16,0$ à 19,1$)$ & 43 à 57 & 18,5 & $(16,1$ à 20,9$)$ & \\
\hline $1940-1954$ & 50 à 64 & 14,2 & $(12,6$ à 15,8$)$ & 58 à 72 & 17,3 & $(15,4$ à 19,1$)$ & a \\
\hline 1928-1939 & 65 à 76 & 6,2 & $(4,5$ à 7,9$)$ & 73 à 84 & 10,0 & $(7,5$ à 12,4$)$ & a \\
\hline
\end{tabular}

Sources : GENACIS, enquête canadienne de 2004-2005; Statistique Canada, ESCC-SM, 2012 (fichier partagé).

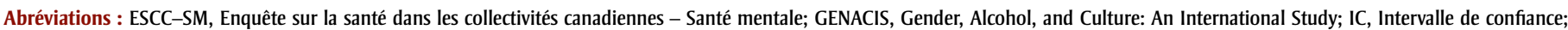
VSdE, violence sexuelle durant l'enfance.

a Écart notable par rapport aux données de 2004-2005. 
femmes nées entre 1940 et 1954 et entre 1928 et 1939 : la prévalence était plus élevée chez les répondants de l'ESCC Santé mentale.

\section{Analyse}

Notre étude des estimations de la prévalence de la VSdE, fondée sur deux grandes études représentatives de la population adulte canadienne, corrobore notre hypothèse que la période à compter de 1993 est à risque de VSdE moindre, que la période 1946-1992 était à risque accru et que la période antérieure à 1946 était également une période à risque moindre. Les preuves sont substantielles pour les femmes. La baisse des estimations de prévalence chez les jeunes hommes ayant répondu à l'ESCC Santé mentale ne témoigne que faiblement de la présence éventuelle d'un léger fléchissement depuis le début des années 1990. Une surveillance accrue est nécessaire pour confirmer cette baisse de la VSE chez les hommes canadiens.

\section{Données probantes et facteurs de baisse}

Nos constatations d'une baisse (ou fléchissement du risque) de la VSE depuis le début des années 1990 concordent avec celles de Finkelhor et ses collaborateurs, lesquelles reposent sur de nombreuses sources de données des États-Unis ${ }^{6,13}$, de même qu'avec les résultats d'une étude représentative menée à l'échelle nationale en Australie ${ }^{22}$. L'examen de données recueillies auprès d'étudiants témoigne également d'une baisse au cours de cette période. À titre d'exemple, à Aotearoa (NouvelleZélande), les signalements de VSE ont connu une baisse entre 2001 et 2007, dont une baisse plus importante chez les hommes $^{23}$. En Colombie-Britannique (Canada), on a constaté un recul entre 1992 et $2003^{24}$. Les données recueillies auprès des autorités de protection de l'enfance dans le cadre de l'Étude canadienne sur l'incidence des signalements de cas de violence et de négligence envers les enfants (ECI) laissent aussi supposer une baisse ${ }^{25,26}$. En dépit des inquiétudes soulevées à propos de la représentativité nationale des estimations issues des données pondérées de l'ECI ${ }^{27}$, ces estimations font état d'une baisse du nombre d'enquêtes sur les cas de violence sexuelle corroborés pour 1000 enfants âgés de 0 à 15 ans, qui est passé de 86 en 1998 à 43 en $2008^{25,26}$. De même, le taux de signalement des agressions sexuelles à la police a culminé en 1993, pour ensuite diminuer progressivement de la fin des années 1990 jusqu'aux années $2000^{12}$.

Finkelhor et Jones ${ }^{9}$ ont étudié de nombreux facteurs susceptibles d'expliquer la baisse de la VSE observée aux États-Unis, et ils ont conclu que celle-ci s'explique vraisemblablement par un faisceau de facteurs. Ils avancent que la prospérité économique, l'augmentation des services de police et la hausse du nombre d'agents d'intervention sociale - les travailleurs sociaux, les intervenants en protection de l'enfance et les spécialistes en pharmacothérapie pour les troubles psychiatriques, entre autres - constituent l'explication la plus plausible. Ils estiment par ailleurs que l'accessibilité accrue à des moyens de contraception efficaces au cours des années précédant la période de baisse de la VSE et durant cette période a conduit à une diminution du nombre de grossesses non désirées et à de meilleurs chances que les enfants grandissent dans des environnements dotés de ressources socioéconomiques suffisantes pour assurer leur protection. La modification des normes et des pratiques a pu également jouer un rôle en ce sens. De surcroît, l'augmentation des niveaux d'instruction pourrait avoir entraîné une sensibilisation accrue à la VSE et à l'importance d'offrir un environnement sécuritaire aux enfants.

Certains facteurs expliquant la baisse de la VSE aux États-Unis ne sont pas nécessairement pertinents dans le contexte canadien. Une étude comparative des facteurs de la baisse générale du crime observé dans les années 1990 au Canada comme aux États-Unis fait par exemple état d'explications quelque peu différentes pour les deux pays ${ }^{28}$. Au Canada, contrairement aux États-Unis, la taille des services de police et le taux d'incarcération ont peu, voire pas du tout, changé pendant la période de baisse. C'est plutôt l'évolution des données démographiques, des taux d'emploi et des valeurs collectives qui est vraisemblablement à l'origine du phénomène ${ }^{28}$. Par ailleurs, certaines explications avancées par Finkelhor et Jones ${ }^{9}$ sont étayées par des sources de données canadiennes, comme la baisse des grossesses chez les adolescentes $^{29}$, l'augmentation des niveaux d'instruction $^{30}$ et les baisses du taux de chômage ${ }^{28}$. La prise d'antidépresseurs a également augmenté au Canada dès le début des années $1990^{31}$. Les parents atteints de troubles mentaux qui bénéficient d'un traitement seraient mieux à même de prendre en charge et de protéger leurs enfants que ceux qui ne bénéficient pas du traitement nécessaire ${ }^{9}$. Enfin, les programmes de prévention de la VSE dans les écoles ainsi que les campagnes de sensibilisation au problème pourraient également avoir eu une incidence positive $\mathrm{e}^{32,33}$.

\section{Données probantes et facteurs d'augmentation du risque}

Les facteurs expliquant la hausse de la VSE après la Seconde Guerre mondiale sont moins évidents. De fait, la rareté des enquêtes effectuées à cette époque et les méthodologies incohérentes employées pour effectuer ces rares enquêtes réduisent la pertinence des résultats datant de cette époque. Par exemple, toute comparaison directe entre l'enquête GENACIS, l'ESCC Santé mentale et une enquête canadienne de 1983 s'avère impossible à cause de méthodologies divergentes ${ }^{34}$. Les résultats de cette enquête corroborent néanmoins notre hypothèse d'une augmentation du risque de la VSE après la Seconde Guerre mondiale. Bien que cette étude ne fournisse que des données limitées sur les groupes d'âge, un rapport connexe indique que la prévalence de la VSdE était deux fois plus élevée chez les répondants de moins de 25 ans que chez ceux de 44 ans et plus ${ }^{14}$. Les répondants de moins de 25 ans au moment de cette étude auraient vécu toute leur enfance dans la période dite à risque accru (entre 1946 et 1992) tandis que les répondants de 44 ans et plus auraient passé la majorité de leur enfance durant la période dite à risque moindre (avant 1946). Les résultats issus d'autres enquêtes indiquent également une augmentation de la VSE durant la période qui a suivi la Seconde Guerre mondiale $e^{3,15,16}$.

Les modifications de la structure familiale survenues après la Seconde Guerre mondiale pourraient avoir contribué à cette hausse de la $\operatorname{VSE}^{14,35}$. Avant la Seconde Guerre mondiale, le divorce était à peu près inexistant au Canada. Il est devenu plus courant après la Seconde Guerre mondiale, et le taux de divorce a culminé dans la foulée des modifications apportées à la Loi sur le divorce à la fin des années $1980^{35}$. Depuis le début des années 1990, le taux de divorce est demeuré relativement stable. Il ressort de plusieurs études réalisées sur la VSE dans les années 1970 et 1980 que les enfants vivant avec un seul parent ou avec un beau-parent couraient un risque plus élevé de vivre de la VSE que ceux vivant avec leurs deux parents biologiques $^{36-38}$, ce qui pourrait potentiellement 
expliquer la hausse de la VSE après la Seconde Guerre mondiale. Les caractéristiques des familles monoparentales et recomposées des années 1990 et ultérieures pourraient différer de celles des familles des années antérieures. Les autres facteurs précités contribuent vraisemblablement la baisse de la VSE au cours de la période de stabilité du taux de divorce, soit au début des années 1990.

\section{Points forts et limites}

Notre étude utilise une nouvelle approche (le concept d' " années-enfants ») pour dégager les tendances de la VSE au Canada à partir des données de deux grandes études représentatives de la population canadienne. L'épidémiologie de cette violence étant difficile à mesurer, notre approche des " années-enfants » pourrait faciliter les travaux à venir car elle permet de dégager les tendances de la VSE en fonction de la prévalence de la VSdE chez les répondants adultes et adolescents aux enquêtes rétrospectives.

Bien que le recours aux enquêtes rétrospectives pour examiner les tendances de la violence sexuelle à l'endroit des enfants permette de combler certaines lacunes attribuables à une sous-déclaration de cette forme de violence dans les données des organismes, il n'en reste pas moins que les données tirées de ces enquêtes ont leurs limites. De fait, si les déclarations faussement positives de VSdE sont plutôt rares dans le cadre d'enquêtes rétrospectives ${ }^{39}$, le taux de déclarations faussement négatives s'avère considérable (entre $40 \%$ et $50 \%$ ) $^{40-42}$. La sous-déclaration est plus courante chez les répondants de sexe masculin ${ }^{42,43}$. Même si les éléments utilisés pour mesurer la VSE dans l'ESCC - Santé mentale et dans l'enquête GENACIS n'ont pas été validés, des éléments de même types ont été employés dans le cadre de l'Enquête sociale générale canadienne ${ }^{12}$ et ces éléments, axés sur le comportement, apportent davantage d'exactitude et de fiabilité que les questions générales et subjectives ${ }^{39}$. Par ailleurs, les deux études excluent les personnes vivant en établissement de même que celles sans domicile fixe, une population au sein de laquelle la VSE est probablement plus répandue.

Les écarts dans les estimations issues de différentes sources de données pourraient découler de méthodologies divergentes plutôt que de véritables différences de prévalence. En effet, les éléments utilisés pour mesurer la VSE ne sont pas les mêmes dans l'enquête GENACIS et dans l'ESCC Santé mentale, les taux de réponse et les modes d'administration non plus. De plus, les participants à l'ESCC - Santé mentale devaient répondre à quatre autres questions sur d'autres formes de mauvais traitements durant l'enfance avant les deux questions sur la VSE, alors que les participants à l'enquête GENACIS n'avaient pas d'entrée en matière équivalente. Malgré ces différences, les comparaisons entre les cohortes ayant la même année de naissance ont permis de dégager des estimations de prévalence similaires pour les trois cohortes les plus jeunes, ce qui laisse penser que les deux études ont mesuré un phénomène analogue. Nous ignorons cependant dans quelle mesure le taux de réponse - plus particulièrement le faible taux de réponse de l'enquête GENACIS - a eu une incidence sur les estimations de prévalence de la VSdE.

Même si la comparaison de prévalence entre groupes d'âge d'une même enquête permet de passer outre à certaines inconsistances méthodologiques entre enquêtes, des contraintes demeurent. La probabilité que les participants disent la vérité varie en fonction de l'âge. Cela signifie qu'ils ne sont pas nécessairement prêts à divulguer leur expérience de VSdE avant qu'un certain nombre d'années ne se soit écoulé après les actes ${ }^{43}$. Quant à la faible prévalence de la VSdE chez les répondants plus âgés, elle pourrait découler, du moins en partie, de la mortalité prématurée des victimes de $\mathrm{VSE}^{44,45}$. Plus les actes de VSE reculent dans le temps, plus les estimations de prévalence biaisées à la baisse augmentent en raison de la mortalité prématurée : les personnes décédées ne peuvent faire partie de l'échantillon et déclarer la VSdE subie. C'est pourquoi la tendance à la hausse de la VSE avant et après la Seconde Guerre mondiale pourrait être surestimée, et la tendance à la baisse depuis 1993, légèrement sous-estimée.

Les tendances séculaires pourraient également avoir une incidence sur la probabilité de la divulgation de VSdE. Les comparaisons entre cohortes ayant la même année de naissance indiquent une prévalence plus forte chez les répondants de l'ESCC Santé mentale que chez ceux de l'enquête GENACIS pour les femmes nées entre 1928 et 1954 et pour les hommes nés entre 1940 et 1954. Ces résultats peuvent provenir d'une plus forte propension chez ces groupes à divulguer la VSdE, peut-être en raison de la couverture médiatique de cas notoires ${ }^{46}$ ou de l'attention que les médias accordent aux cas de violence sexuelle. Il est à noter à ce propos que l'attention fréquente ou intense que les médias accordent à cette question peut amener la population à croire, en dépit des tendances réelles de son incidence, que la VSE est en hausse. Par ailleurs, même si la propension à divulguer de tels actes est susceptible d'augmenter avec l'âge, la faible prévalence observée chez les répondants âgés peut découler de leur réticence persistante à divulguer cette forme de violence car ils sont nés à une époque où le statut de victime sexuelle était susceptible d'être davantage stigmatisé.

Compte tenu de ces contraintes, de la nouveauté du concept d'année-enfant et de la rareté des analyses antérieures des données canadiennes, nous recommandons la duplication de notre étude, au Canada comme à l'étranger, afin d'apporter des preuves supplémentaires d'un déclin réel de la VSE.

\section{Conclusion}

Les signes du recul de la VSE au Canada depuis le début des années 1990, qui coïncide avec une baisse semblable aux ÉtatsUnis au cours de la même période, sont encourageants. Cependant, en 2012, encore $6 \%$ des femmes et $2 \%$ des hommes de 18 à 24 ans ont indiqué avoir subi de la VSdE, données qui ne reflètent probablement pas la véritable ampleur du problème. Poursuivre les travaux de surveillance continue de la VSE est donc essentiel. Les études rétrospectives de population associées à de solides composantes psychométriques et fondées sur des méthodologies cohérentes constituent un moyen efficace de dégager les tendances de la VSE. Utiliser les sources de données contemporaines issues d'études menées auprès d'élèves et d'étudiants vont permettre de dresser un portrait encore plus exact de la situation. Il est en effet essentiel de réunir des données de grande qualité pour évaluer efficacement les programmes d'intervention visant à éliminer la violence sexuelle envers les enfants canadiens. Les effets préjudiciables à long terme de la VSE montrent bien à quel point il est important de poursuivre sur cette lancée, pour passer d'un risque faible à un risque nul - aucun laisser-aller n'est tolérable. 


\section{Remerciements}

Les données recueillies dans le cadre de l'enquête Gender, Alcohol and Culture: An International Study (GENACIS) nous ont été offertes par l'Institut de recherche sociale de l'Université York. L'enquête a été réalisée au nom du Centre de toxicomanie et de santé mentale (CAMH). Le volet canadien de l'enquête GENACIS a été financé grâce à une subvention de fonctionnement offerte par les Instituts de recherche en santé du Canada (IRSC). Le CAMH, l'Institut de recherche sociale et les IRSC ne sont aucunement responsables des analyses ni des interprétations présentées dans cet article.

Les auteures souhaitent remercier Kathryn Wilkins de son aide à exprimer le plus clairement possible les constructions complexes utilisées dans ces analyses.

\section{Références}

1. Gilbert R, Widom CS, Browne K, Fergusson D, Webb E, Janson S. Burden and consequences of child maltreatment in high-income countries. Lancet. 2009;373(9657):68-81. doi : 10.1016/S0140-6736(08)61706-7.

2. Maniglio R. The impact of child sexual abuse on health: a systematic review of reviews. Clin Psychol Rev. 2009; 29(7):647-657. doi : 10.1016/j.cpr.2009 .08 .003 .

3. Finkelhor D. Current information on the scope and nature of child sexual abuse. Future Child. 1994;4(2):31-53. doi : $10.2307 / 1602522$.

4. Lindsey D. Mandated reporting and child abuse fatalities: requirements for a system to protect children. Soc Work Res. 1994;18(1):41-54.

5. Sedlack AJ, Broadhurst DD. Third National Incidence Study of Child Abuse and Neglect. Washington (DC) : US Department of Health and Human Services; 1996.

6. Finkelhor D, Saito K, Jones L. Updated trends in child maltreatment, 2013. Durham (NH): Crimes against Children Research Center; 2015.
7. Almeida J, Cohen AP, Subramanian SV, Molnar BE. Are increased worker caseloads in state child protective service agencies a potential explanation for the decline in child sexual abuse? A multilevel analysis. Child Abuse Negl. 2008;32(3):367-375. doi : 10.1016 /j.chiabu.2007.08.003.

8. Finkelhor D, Jones LM. Explanations for the decline in child sexual abuse cases. Juvenile Justice Bulletin. Washington (DC) : Office of Juvenile Justice and Delinquency Prevention; 2004.

9. Finkelhor D, Jones LM. Why have child maltreatment and child victimization declined? J Soc Issues. 2006;62(4):685-716. doi : 10.1111/j.1540 $-4560.2006 .00483 . x$.

10. Land KC, Lamb VL, Kahler Mustillo S. Child and youth well-being in the United States, 1975-1998: some findings from a new index. Soc Indic Res. 2001;56(3):241-320. doi : 10.1023/A :1012485315266.

11. Afifi TO, MacMillan HL, Taillieu $\mathrm{T}$ et collab. Relationship between child abuse exposure and reported contact with child protection organizations: results from the Canadian Community Health Survey. Child Abuse Negl. 2015;46:198-206. doi : 10.1016/j.chiabu .2015.05.001.

12. Brennan S, Taylor-Butts A. Les agressions sexuelles au Canada 2004 et 2007. Série de profils du Centre canadien de la statistique juridique. Ottawa (Ont.) : Statistique Canada; 2008. [no 85F0033M au catalogue].

13. Finkelhor D, Jones LM. Have sexual abuse and physical abuse declined since the 1990s? Durham (NH): Crimes against Children Research Center; 2012.

14. Bagley C. Child sexual abuse in Canada: further analysis of the 1983 national survey. Ottawa (Ont.) : Santé et Bienêtre Canada; 1988.

15. Leventhal JM. Have there been changes in the epidemiology of sexual abuse of children during the 20th century? Pediatrics. 1988;82(5):766-773.
16. Wyatt GE, Loeb TB, Solis B, Carmona JV, Romero, G. The prevalence and circumstances of child sexual abuse: changes across a decade. Child Abuse Negl. 1999;23(1):45-60. doi : 10.1016 /S0145-2134(98)00110-0.

17. Feldman W, Feldman E, Goodman JT et collab. Is childhood sexual abuse really increasing in prevalence? An analysis of the evidence. Pediatrics. 1991;88(1):29-33. doi : 10.1016/s0890 $-8567(10) 80016-4$.

18. Gorey KM, Leslie DR. The prevalence of child sexual abuse: integrative review adjustment for potential response and measurement biases. Child Abuse Negl. 1997;21(4):391-398. doi : 10.1016/s0145-2134(96)00180-9.

19. Statistique Canada. Guide de l'utilisateur de l'Enquête sur la santé dans les collectivités canadiennes (ESCC) - Santé mentale. Ottawa (Ont.) : Statistique Canada; 2013.

20. Graham K, Bernards S. Canada: alcohol and partner physical aggression in the 10 provinces. Dans : Graham K, Bernards S, Munné M, Wilsnack S (dir.), Unhappy Hours: Alcohol and Physical Partner Aggression in the Americas (p. 90-108). Washington (DC) : Organisation panaméricaine de la santé; 2008.

21. Graham K, Massak A. Alcohol consumption and the use of antidepressants. CMAJ. 2007;176(5):633-637. doi : 10.1503/cmaj.060446.

22. Dunne MP, Purdie DM, Cook MD, Boyle FM, Najman JM. Is child sexual abuse declining? Evidence from a population-based survey of men and women in Australia. Child Abuse Negl. 2003;27(2):141-152. doi : 10.1016/s0145 $-2134(02) 00539-2$.

23. Denny SJ, Grant S, Utter J et collab. Health and well-being of young people who attend secondary school in Aotearoa, New Zealand: what has changed from 2001 to 2007? J Paediatr Child Health. 2011;47(4):191-197. doi : 10.1111/j.1440-1754.2010.01945.x.

24. Tonkin RS. British Columbia Youth Health Trends: A Retrospective, 19922003. Vancouver (C.-B.) : McCreary Centre Society; 2005. 
25. Agence de la santé publique du Canada. Étude canadienne sur l'incidence des signalements de cas de violence et de négligence envers les enfants - 2008 : données principales. Ottawa (Ont.) : Agence de la santé publique du Canada; 2010.

26. Trocmé N, MacLaurin B, Fallon B et collab. Étude canadienne sur l'incidence des signalements de cas de violence et de négligence envers les enfants (EIC) : Rapport final. Ottawa (Ont.) : Ministre de Travaux publics et Services gouvernementaux Canada; 2001.

27. Dumais J. Comparison and assessment of CIS 1998, 2003 and 2008 designs. Ottawa (Ont.) : Statistique Canada; 2010. Rapport non publié.

28. Ouimet M. Explaining the American and Canadian crime "drop" in the 1990s. Can J Criminol. 2002;44(1): 33-50.

29. McKay A. Trends in Canadian national and provincial/territorial teen pregnancy rates 2001-2010. Can J Hum Sex. 2013;21(3-4):161-175.

30. Statistique Canada, Conseil des ministres de l'éducation, Canada. Indicateurs de l'éducation au Canada : une perspective internationale. Ottawa (Ont.) : Statistique Canada; 2014. [no 81-604-X au catalogue].

31. Hemels ME, Koren G, Einarson TR. Increased use of antidepressants in Canada: 1981-2000. Ann Pharmacother. 2002;36(9):1375-1379. doi : 10.1345 /aph.1a331.

32. MacMillan HL, Wathen CN, Barlow J, Fergusson DM, Leventhal JM, Taussig HN. Interventions to prevent child maltreatment and associated impairment. Lancet. 2009;373(9659):250-266. doi : 10.1016/S0140-6736(08)61708-0.

33. Walsh K, Zwi K, Woolfenden S, Shlonsky A. School-based education programmes for the prevention of child sexual abuse. Cochrane Database Syst Rev. 2015;4:CD004380. doi : 10.1002 /14651858.CD004380.pub3.

34. Badgley RF, Allard HA, McCormick N et collab. Infractions sexuelles à l'égard des enfants (Rapport Badgley). Ottawa (Ont.) : ministère des Approvisionnements et Services; 1984.
35. Milan A. État matrimonial : aperçu, 2011. Rapport sur l'état de la population du Canada. Ottawa (Ont.) : Statistique Canada : 2013 [no 91-209-X au catalogue].

36. Daly M, Wilson M. Child abuse and other risks of not living with both parents. Ethol Sociobiol. 1985;6(4):197-210.

37. Fleming J, Mullen P, Bammer G. A study of potential risk factors for sexual abuse in childhood. Child Abuse Negl. 1997;21(1):49-58. doi : 10.1016 /s0145-2134(96)00126-3.

38. Giles-Sims, J. Current knowledge about child abuse in stepfamilies. Marriage Fam Rev. 1997;26:215-230.

39. Hardt J, Rutter M. Validity of adult retrospective reports of adverse childhood experiences: review of the evidence. J Child Psychol Psychiatry. 2004;45(2):260-273. doi : 10.1111/j .1469-7610.2004.00218.x.

40. Fergusson DM, Horwood LJ, Woodward LJ. The stability of child abuse reports: a longitudinal study of the reporting behaviour of young adults. Psychol Med. 2000;30(3):529-544. doi : 10.1017 /s0033291799002111.

41. Scott KM, Smith DR, Ellis PM Prospectively ascertained child maltreatment and its association with DSM-IV mental disorders in young adults. Arch Gen Psychiatry. 2010; 67(7):712-719.

42. Widom CS, Morris S. Accuracy of adult recollections of childhood victimization: Part 2. Childhood sexual abuse. Psychol Assess. 1997;9(1):3446. doi : 10.1037/1040-3590.9.1.34.

43. Hébert M, Tourigny M, Cyr M, McDuff P, Joly J. Prevalence of childhood sexual abuse and timing of disclosure in a representative sample of adults from Quebec. Rev. can. psychiatrie. 2009;54(9):631-636. doi : 10.1177 /070674370905400908.

44. Brown DW, Anda RF, Tiemeier $\mathrm{H}$ et collab. Adverse childhood experiences and the risk of premature mortality. Am J Prev Med. 2009;37(5):389-396. doi : 10.1016/j.amepre.2009.06.021
45. Felitti VJ, Anda RF, Nordenberg D et collab. Relationship of childhood abuse and household dysfunction to many of the leading causes of death in adults. The Adverse Childhood Experiences (ACE) Study. Am J Prev Med. 1998;14(4):245-258. doi : 10.1016/s0749 $-3797(98) 00017-8$.

46. Weatherred JL. Child sexual abuse and the media: a literature review. J Child Sex Abus. 2015;24(1):16-34. doi : 10.1080/10538712.2015.976302. 